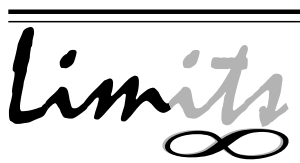

J. Math. and Its Appl.

ISSN : 1829-605X

Vol. 3, No. 2, Nov 2006, 57-66

\title{
Disturbance Rejection Problem with Stability By Static Output Feedback Of Linear Continuous Time System
}

\author{
Soleha \\ Mathematics Department \\ Institut Technology of Sepuluh Nopember Surabaya \\ sehamaniz_07@yahoo.com
}

\begin{abstract}
Disturbance rejection problem with stability by static output feedback of linear-time invariant continuous time system is solvable if there is found a static output feedback control law, $u(t)=K y(t)$ (if possible), such that disturbance $q(t)$ has no influence in controlled output $z(t)$. So, it is needed the necessary and sufficient condition disturbance rejection problem is solvable. By using the definition and characteristics of $(A, B)$-invariant subspace, and $(C, A)$-invariant subspace, then it will be find the necessary and sufficient condition disturbance rejection problem of that system will be solved if and only if maximal element of a set of $(C, A)$-invariant is an $(A$, $B$ )-invariant subspace that internally stabilizable and externally stabilizable.
\end{abstract}

Key Words: Linear system; Disturbance rejection problem; (A,B)invariant subspace; ( $C, A)$-invariant subspace 


\section{Introduction}

In system theory, there are many system models. Once is a linear, continuous-time system described by:

$$
\begin{aligned}
\dot{x}(t) & =A x(t)+B u(t) \\
y(t) & =C x(t)
\end{aligned}
$$

where $x \in R^{n}$ is the state, $u \in R^{m}$ is the control input, $y \in R^{h}$ is the output. $A \in R^{n}$ is a matrix, $B \in R^{n \times m}$ is a matrix, and $C \in R^{h \times n}$ is a matrix. If that system has a disturbance, we can make the model of the system likes:

$$
\begin{aligned}
\dot{x}(t) & =A x(t)+B u(t)+E q(t) \\
y(t) & =C x(t)
\end{aligned}
$$

where $q \in R^{q}$ is the disturbance input, and $E \in R^{n \times q}$ is a matrix. When there is a disturbance in a system, it may cause deviation between the expecting result and the obtained output. Therefore, it needs a feedback control which becomes control rule which gives information from output deviation and entry that information as a new input, then all the deviations from the expecting result can be corrected. A solution of Disturbance Rejection Problem with stability by Static Input Feedback, $u(t)=F x(t)$ has been recently proposed in [9]. When the state vector is not available for measurement, [3] are led to consider the Disturbance Rejection Problem by Static Output Feedback, $u(t)=K y(t)$ with the system is:

$$
\begin{aligned}
\dot{x}(t) & =A x(t)+B u(t)+E q(t) \\
y(t) & =C x(t) \\
z(t) & =D x(t)
\end{aligned}
$$

where $y \in R^{h}$ is measured output $z \in R^{z}$ is the controlled output, and $D \in R^{z \times n}$ is a matrix . For next, the system in 2 will be called $(A, B, C, D, E)$ system. In this paper will be studied the solution of Disturbance Rejection with Stability by Output Static Feedback related to the system with the system class is:

$$
\gamma^{*} \subset \operatorname{Ker}(D)+S_{*}
$$

Where $\gamma^{*}=$ maximal $(A, B)$ invariant subspace contained in $\operatorname{Ker}(D)$, and $S_{*}=$ minimal $(C, A)$ invariant subspace containing Image $(E)$. 


\section{Preliminaries}

Consider $(A, B, C, D, E)$ system, if we give Static Output Feedback, $u(t)=$ $K y(t)$, where $K \in R^{m \times h}$ is a matrix, then system in (1) becomes:

$$
\begin{aligned}
& \dot{x}(t)=A x(t)+B K y(t)+E q(t) \\
& \dot{x}(t)=A x(t)+B K C x(t)+E q(t)
\end{aligned}
$$

The solution of the equation above by using linear differential equation if $\mathrm{x}(0)=$ $x_{0}$ is:

$$
\begin{aligned}
& x(t)=x_{0} e^{(A+B K C) t}+\int_{0}^{t} e^{(A+B K C) t} E q(s) d s \\
& z(t)=D x_{0} e^{(A+B K C) t}+D \int_{0}^{t} e^{(A+B K C) t} E q(s) d s
\end{aligned}
$$

The goal of the Disturbance Rejection Problem by Static Output Feedback is to determine, if possible, a static output feedback control law, $u(t)=K y(t)$, such that the transfer matrix from disturbance $q$ to controlled output $z$ is null, or:

$$
D \int_{0}^{t} e^{(A+B K C) t} E q(s) d s=0
$$

\section{Invariant Subspaces}

$(A, B)$-invariant subspace and $(C, A)$-invariant are basic in the study of this problem. In this section, we bring definition and characteristic both of them.

Definition 2.1 [3] Consider the pair (A, B) related to the system (1). A subspace $\gamma \subset R^{n}$ is said to be $(A, B)$ invariant if $A \gamma \subset \gamma+B$.

We can show that, equivalently, $\gamma \subset R^{n}$ is $(A, B)$ invariant if and only if there exist a matrix $F \in R^{m \times n}$ such that $(A+B F) \gamma \subset \gamma$.

We can also show that the set of all $(A, B)$-invariant subspace contained in $\operatorname{Ker}(D)$ has a maximal element:

$\gamma^{*}=$ maximal $(A, B)$-invariant subspace contained in $\operatorname{Ker}(D)$.

Definition 2.2 [3] Consider the pair (C, A) related to the system (1). A subspace $S \subset R^{n}$ is said to be $(C, A)$-invariant if $A(S \cap \operatorname{Ker}(C)) \subset S$.

Equivalently, $S$ is $(C, A)$-invariant if and only if there exists a matrix $G \in R^{n \times h}$ such that $(\mathrm{A}+\mathrm{GC}) S \subset S$. 
The set of all $(C, A)$-invariant subspaces containing $\operatorname{Im}(E)$ which is defined by $\xi(C, A ; \operatorname{Im}(E)$ has a minimal element:

$S_{*}=$ minimal $(C, A)$-invariant subspaces containing $\operatorname{Im}(E)$.

From the above definition we can prove this theorem:

Theorem 2.3 [1] (C, A)-invariant is closed under subspace addition.

Gathering definition and characteristic of $(A, B)$ invariant and $(C, A)$ invariant, we can make a subspace $V$ which is $(A, B)$ invariant and $(C, A)$-invariant become a new form of invariant subspace that will be explained in this following theorem:

Theorem 2.4 [1] Let $A \in R^{n}$ is a matrix, $B \in R^{n \times m}$ is a matrix, $C \in R^{h \times n}$ is a matrix and $K \in R^{m \times h}$ is a matrix. A subspace $V$ is $(A+B K C)$-invariant if and only if $V$ is $(A, B)$-invariant and $(C, A)$-invariant.

\section{Internally Stabilizable and Externally Stabilizable}

An invariant subspace of a matrix $A \in R^{n}$ always has linear transformation. From that, we can classification an invariant subspace becoming internally stabilizable or externally stabilizable, which will be explained in these following theorem and definition:

Theorem 2.5 [1] Let $A \in R^{n}$ is a matrix, and subspace $J \in R^{n}$ is an A-invariant, then there exist linear transformation $T$ such that

$$
A^{\prime}=T^{-1} A T=\left(\begin{array}{cc}
A_{11}^{\prime} & A_{12}^{\prime} \\
0 & A_{22}^{\prime}
\end{array}\right)
$$

with $A_{11}^{\prime} \in R^{h \times h}$ is a matrix and dimension of $J=h$.

Definition 2.6 [1] Let subspace $J \in R^{n}$ is an A-invariant.

1. $J$ is called internally stabilizable if the differences eigen value of $A_{11}^{\prime}$, denoted by $\lambda_{1}, \lambda_{2}, \ldots, \lambda_{k}(k \leq h)$ implies $\operatorname{Re}\left(\lambda_{i}\right)<0$ for $i=1,2, \ldots, k$

2. $J$ is called externally stabilizable if the differences eigen value of $A_{22}^{\prime}$, denoted by $\lambda_{1}, \lambda_{2}, \ldots, \lambda_{k}(k \leq(n-h))$ implies $\operatorname{Re}\left(\lambda_{i}\right)<0$ for $i=1,2, \ldots, k$

We can use this following theorem to look for determinant of $\mathrm{A}^{\prime}$ in (3).

Theorem 2.7 [1] Let $A \in R^{n}$ is a matrix, then $\operatorname{det}\left(A^{\prime}\right)=\operatorname{det}(A)=\operatorname{det}\left(A_{11}^{\prime}\right)$. $\operatorname{det}\left(A_{22}^{\prime}\right)$. 
Consider now the following class of subspaces

$$
\langle A+B K C \mid \operatorname{Im} E\rangle=\operatorname{Im}(E)+(A+B K C) \operatorname{Im}(E)+\cdots+(A+B K C)^{n-1} \operatorname{Im}(E)
$$

where $(A+B K C)^{n-1}$ is composition function as n-1 times.

Definition 2.8 [9] Disturbance rejection problem at ( $A, B, C, D, E)$ system with stability by static output feedback can be solved if there exist a matrix $K \in R^{m \times h}$ such that $\langle A+B K C \mid \operatorname{Im} E\rangle \subset \operatorname{Ker}(D)$ and $\sigma(A+B K C) \subset C_{g}$ where $\sigma(A+B K C)$ represents the set of $(A+B K C)$ 's eigen value, and $C_{g}=\left\{s ; s \in C, \operatorname{Re}\left(\lambda_{s}\right)<0\right\}$

\section{Class of System: Self Hidden}

In this paper, we want to establish the geometric condition which defined the class of system. The class is satisfying condition that is explained in this following definition:

Definition $2.9[3] S \in \xi(C, A ; \operatorname{Im}(E)$ is said to be self hidden with respect to $\operatorname{Im}(E)$ if $S \subset S_{*}+\operatorname{Ker}(C)$

\section{Discussion}

The preceding Theorem establishes necessary and sufficient condition for existence of static output feedback which solves Disturbance Rejection with Stability by Output Static Feedback with the system class is $\gamma^{*} \subset \operatorname{Ker}(C)+S_{*}$.

The first theorem that we have to prove is showing that there is a subspace which become $(A, B)$-invariant and also $(C, A)$ invariant likes in this following theorem

Theorem 3.1 $\langle A+B K C \mid \operatorname{Im} E\rangle$ is $(A, B)$ and $(C, A)$-invariant.

\section{Proof:}

For arbitrary $h \in(A+B K C)\langle A+B K C \mid \operatorname{Im} E\rangle$, we have $h=(A+B K C) x$, where $x \in\langle A+B K C \mid \operatorname{Im} E\rangle$. Then there exist $w_{1}, w_{2}, \ldots, w_{n} \in \operatorname{Im}(E)$ such that

$$
\begin{aligned}
x= & w_{1}+(A+B K C) w_{2}+\ldots+(A+B K C)^{n-1} w_{n} \\
h= & -p_{n}\left(w_{n}\right)+(A+B K C)\left(w_{1}-p_{n-1} w_{n}\right)+(A+B K C)^{2}\left(w_{2}-p_{n-2} w_{n}\right) \\
& +\cdots+(A+B K C)^{n-1}\left(w_{n-1}-p_{1} w_{n}\right)
\end{aligned}
$$

Since $\operatorname{Im}(E)$ subspace $R^{n}$, we get

$$
-p_{n}\left(w_{n}\right),\left(w_{1}-p_{n-1} w_{n}\right),\left(w_{2}-p_{n-2} w_{n}\right), \ldots,\left(w_{n-1}-p_{1} w_{n}\right) \in \operatorname{Im}(E)
$$


Therefore we have $(A+B K C)\langle A+B K C \mid \operatorname{Im} E\rangle \subset\langle A+B K C \mid \operatorname{Im} E\rangle$

From Definition 2.1 and Theorem 2.4, $\langle A+B K C \mid \operatorname{Im} E\rangle$ is $(A, B)$ and $(C$, A)-invariant.

Before we prove the last theorem, we need to find necessary and sufficient condition of disturbance rejection problem which not contain a system class which is included in this following theorem:

Theorem 3.2 Disturbance rejection problem at (A, B, C, D, E) system with stability by static output feedback can be solved if and only if there exist $V$ subspace and a matrix $K \in R^{m x h}$ such that

1. $\operatorname{Im}(E) \subset V \subset \operatorname{Ker}(D)$

2. $(A+B K C) V \subset V$

3. $\sigma(A+B K C) \subset C_{g}$

\section{Proof:}

Necessity:

1. By using Definition 2.8 there exists a matrix $K \in R^{m x h}$ such that $\langle A+$ $B K C|\operatorname{Im} E\rangle \subset \operatorname{Ker}(\mathrm{D})$ and $\sigma(A+B K C) \subset C_{g}$.

We take $V=\langle A+B K C \mid \operatorname{Im} E\rangle$. For arbitrary $p \in \operatorname{Im}(E)$, there exists $z \in R^{q}$ such that $p=E(z)$.

$p=E(z)=E(z)+(A+B K C) \overline{0}+(A+B K C)^{2} \overline{0}+\ldots+(A+B K C)^{n-1}$ $\overline{0}$. Because $\mathrm{E}(0)=$, then $p \in\langle A+B K C \mid \operatorname{Im} E\rangle$. So $\operatorname{Im}(E) \subset\langle A+$ $B K C|\operatorname{Im} E\rangle=V$. By Definition 2.8 we get $\langle A+B K C \mid \operatorname{Im} E\rangle \subset \operatorname{Ker}(D)$, so $\operatorname{Im}(E) \subset V \subset \operatorname{Ker}(D)$.

2. From the Theorem 3.1, we get

$$
(A+B K C)(\langle A+B K C \mid \operatorname{Im} E\rangle=\mathrm{V})(\subset\langle A+B K C \mid \operatorname{Im} E\rangle=\mathrm{V})
$$

3. from the Definition 2.8 we get $\sigma(A+B K C) \subset C_{g}$

Sufficiency: Because $\operatorname{Im}(E) \subset V$, then

$$
\left.\begin{array}{l}
\operatorname{Im}(E)+(A+B K C) \operatorname{Im}(E)+ \\
(A+B K C)^{2} \operatorname{Im}(E)+\ldots+ \\
(A+B K C)^{n-1} \operatorname{Im}(E)
\end{array}\right\} \subset\left\{\begin{array}{l}
V+(A+B K C) V+ \\
(A+B K C)^{2} V+\ldots+ \\
(A+B K C)^{n-1} V
\end{array}\right.
$$

or

$$
\langle A+B K C \mid I m E\rangle \subset\langle A+B K C \mid V\rangle
$$


For arbitrary $g \in\langle A+B K C \mid \operatorname{Im} E\rangle$, there exist $v_{1}, v_{2}, v_{3}, \ldots, v_{n} \in V$ such that $g=v_{1}+(A+B K C) v_{2}+(A+B K C)^{2} v_{3}+\ldots+(A+B K C)^{n-1} v_{n}$. Because $(A+B K C) V \subset V$, then there exist $w_{1}, w_{2}, w_{3}, \ldots, w_{n} \in V$ such that $g=v_{1}+w_{1}+w_{2}+w_{3}+\ldots+w_{n} \in V$. So

$$
\langle A+B K C \mid V\rangle \subset V .
$$

Next, let $v \in V$. Because $V$ is a subspace and $(A+B K C)$ is linear transformation, there exists $0 \in V$, so $v$ can be wrote as:

$v=v+(A+B K C) 0+(A+B K C)^{2} 0+\ldots+(A+B K C)^{n-1} 0$.

So $v \in\langle\mathrm{A}+\mathrm{BKC} \mid \mathrm{V}\rangle$ or

$$
V \subset\langle A+B K C \mid V\rangle
$$

From (4), (5), and (6) we get

$$
\langle A+B K C \mid \operatorname{Im} E\rangle \subset\langle A+B K C \mid V\rangle=V \subset \operatorname{Ker}(D)
$$

then $\langle A+B K C \mid \operatorname{Im}(E)\rangle \subset \operatorname{Ker}(D)$, or disturbance rejection problem at $(A$, $B, C, D, E)$ system with stability by static output feedback can be solved.

Suppose $\Psi(C, A ; \operatorname{Im}(E))$ represent the set of $(C, A)$-invariant subspace containing $\operatorname{Im}(E)$ which are self hidden or we can wrote as:

$\Psi(C, A ; \operatorname{Im}(E))=\left\{S ;(A+G C) S \subset S, S \supset \operatorname{Im}(E)\right.$ and $\left.S \subset \operatorname{Ker}(C)+S_{*}\right\}$

Based on Theorem 2.3 we find that $(C, A)$-invariant is closed under subspace addition. We also can prove that $(\Psi(C, A$; $\operatorname{Im}(E))$ is closed under subspace addition

Suppose $\Gamma(C, A ; \operatorname{Im}(E))$ represent the set of $(C, A)$-invariant subspace containing $\operatorname{Im}(E)$ and contained in $\gamma^{*}$ or it is can be wrote as:

$\Gamma(C, A ; \operatorname{Im}(E))=\left\{S: S \in \xi\left(C, A ; \operatorname{Im}(E)\right.\right.$ and $\left.S \subset \gamma^{*}\right\}$

Based on above sentences we find that $(C, A)$ invariant is closed under subspace addition.

We also can prove if $\gamma^{*} \subset \operatorname{Ker}(C)+S_{*}$ implies $\Gamma(C, A ; \operatorname{Im}(E))$ is closed under subspace addition.

Based on Definition 2.2, we find that set of all $(C, A)$-invariant subspaces containing $\operatorname{Im}(E)$ has a minimal element. In this section, we also can prove that $\Gamma(C, A ; \operatorname{Im}(E))$ also has a maximal element if $\gamma^{*} \subset \operatorname{Ker}(C)+S_{*}$ which is:

$$
S^{*}=\max S \in \Gamma(C, A ; \operatorname{Im}(E))
$$


The last theorem we need to prove is necessary and sufficient condition of disturbance rejection problem at $(A, B, C, D, E)$ system with the system class is $\gamma^{*} \subset \operatorname{Ker}(C)+S_{*}$ with stability by static output feedback can be solved which is mentioned in following theorem:

Theorem 3.3 Disturbance rejection problem at $(A, B, C, D, E)$ system with the system class is $\gamma^{*} \subset \operatorname{Ker}(C)+S_{*}$ with stability by static output feedback can be solved if and only if $S^{*}$ is an $(A, B)$-invariant subspace that internally stabilizable and externally stabilizable.

\section{Proof:}

Necessity: There exists matrix $K \in R^{m \times h}$ and subspace $S$ implies $S_{*}<S<\gamma^{*}$ and $(A+B K C) S \subset S$. This is guarantying that $\Gamma(C, A ; \operatorname{Im}(E))$ is not empty, then there exist maximal element $S^{*}$. Because $S \subset S^{*}$ and $S^{*}$ is an $(C, A)$-invariant, then $(A+B K C) S \subset(A+B K C) S^{*} \subset S^{*}$. It follow based on Theorem 2.4 that $S^{*}$ is an $(A, B)$-invariant.

Because $\sigma(A+B K C) \subset C_{g}$, then based on Definition 2.8, $\operatorname{Re}\left(\lambda_{i}\right)<0$ for $i=$ $1,2, \ldots, k(k \leq n)$. Suppose $A+B K C=M$, then by Theorem 2.4 there exist $T^{-1}$ and $T$ implies

$$
M^{\prime}=T^{-1} M T=\left(\begin{array}{cc}
M_{11}^{\prime} & M_{12}^{\prime} \\
0 & M_{22}^{\prime}
\end{array}\right)
$$

Therefore,

$$
\begin{aligned}
\operatorname{det}\left(\lambda I-M^{\prime}\right) & =\operatorname{det}(\lambda I-M) \\
& =\operatorname{det}\left(\begin{array}{cc}
\lambda I_{h}-M_{11}^{\prime} & -M_{12}^{\prime} \\
0 & \lambda I_{n-h}-M_{22}^{\prime}
\end{array}\right) \\
& =\operatorname{det}\left(\lambda I_{h}-M_{11}^{\prime}\right) \times \operatorname{det}\left(\lambda I_{n-h}-M_{22}^{\prime}\right)
\end{aligned}
$$

Because $\operatorname{Re}\left(\lambda_{i}\right)<0$ for $i=1,2, \ldots, k(k \leq n)$, then $\operatorname{Re}\left(\lambda_{i}\right)<0$ for $i=1,2$, $\ldots, k(k \leq h)$ and $\operatorname{Re}\left(\lambda_{i}\right)<0$ for $i=1,2, \ldots, k(k \leq(n-h))$, therefore $\operatorname{Re}\left(\lambda_{i}\right)<$ 0 for $i=1,2, \ldots, k(k \leq h))$ in sub matrix $M_{11}^{\prime}$ or $\left((\mathrm{A}+\mathrm{BKC})^{\prime} 11\right)$ and $\operatorname{Re}\left(\lambda_{i}\right)$ $<0$ for $i=1,2, \ldots, k(k \leq(n-h))$ in sub matrix $M_{22}^{\prime}$ or $\left((\mathrm{A}+\mathrm{BKC})^{\prime}{ }_{22}\right)$.

It follows based on Definition 2.6, we get $S^{*}$ is a subspace that internal stabilizable and externally stabilizable. Therefore $S^{*}$ is an $(A, B)$-invariant subspace that internally stabilizable and externally stabilizable.

Sufficiency: Suppose $S^{*}$ is an $(A, B)$-invariant. Because $S^{*}$ maximal element in $S \in \Gamma(\mathrm{C}, \mathrm{A} ; \operatorname{Im}(\mathrm{E}))$ then $\operatorname{Im}(E) \subset S_{*} \subset S \subset S^{*} \subset \gamma^{*} \subset \operatorname{Ker}(D)$ 
or $\operatorname{Im}(E) \subset S^{*} \subset \operatorname{Ker}(\mathrm{D})$.

Because $S^{*}$ is an $(A, B)$-invariant and $(C, A)$-invariant then based on Theorem $2.4 S^{*}$ is an $(A+B K C)$-invariant, which is implies based on Definition 2.1 $(A+B K C) S^{*} \subset S^{*}$.

Because $S^{*}$ is an $(\mathrm{A}+\mathrm{BKC})$-invariant that internally stabilizable then sub matrix $\left(M_{11}^{\prime}\right)$ in $(7)$ implies $\operatorname{Re}\left(\lambda_{i}\right)<0$ for $\left.i=1,2, \ldots, k(k \leq h)\right)$. Because $S^{*}$ is an $(A+B K C)$-invariant that externally stabilizable then sub matrix $\left(M_{22}^{\prime}\right)$ in (7) implies $\operatorname{Re}\left(\lambda_{i}\right)<0$ for $i=1,2, \ldots, k(k \leq(n-h))$. Based on (8) implies:

$\operatorname{det}\left(\lambda I-M^{\prime}\right)=\operatorname{det}(\lambda I-M)=\operatorname{det}\left(\lambda I_{h}-M_{11}^{\prime}\right) \times \operatorname{det}\left(\lambda I_{n-h}-M_{22}^{\prime}\right)$

Therefore if $\operatorname{Re}\left(\lambda_{i}\right)<0$ for $i=1,2, \ldots, k(k \leq h)$ and $\operatorname{Re}\left(\lambda_{i}\right)<0$ for

$i=1,2, \ldots, k(k \leq(n-h))$ implies $\operatorname{Re}\left(\lambda_{i}\right)<0$ for $i=1,2, \ldots, k(k \leq n)$

Therefore based on Definition 2.8, $\sigma(A+B K C) \subset C_{g}$.

Because $\operatorname{Im}(E) \subset S^{*} \subset \operatorname{Ker}(\mathrm{D}),(A+B K C) S^{*} \subset S^{*}$ and $\sigma(A+B K C) \subset C_{g}$, then disturbance rejection problem at $(A, B, C, D, E)$ system with the system class is $\gamma^{*} \subset \operatorname{Ker}(D)+S_{*}$ with stability by static output feedback can be solved.

\section{Conclusion}

Disturbance rejection problem at $(A, B, C, D, E)$ system with the system class is $\gamma^{*} \subset \operatorname{Ker}(D)+S_{*}$ with stability by static output feedback can be solved if and only if $S^{*}$ is an $(A, B)$-invariant subspace that internally stabilizable and externally stabilizable.

\section{References}

[1] Basile G. and Marro G. Controlled and Conditioned Invariants in Linear System Theory. Prentice-Hall, 1992

[2] Burghes D.N. and Graham A. Introduction to control Theory Including Optimal Control. John Wiley and Sons. Inc, New York, 1980

[3] Dorea C.E.T. and Milani B.E.A. Disturbance Decoupling via Static Output Feedback for Particular Classes of Linear Systems, 2000 (http:// www.univ perp.r/mtns2000/articles/B221.pdf)

[4] Decarlo R.A. Linear System: A State Variable Approach with Numerical Implementation. Prentice-Hall, New York, 1989 [ 
[5] Goldberg J.L. Matrix Theory with Application. Mc Graw-Hall. Inc, New York, 1991

[6] Jacob B. Linear Algebra. W.H. Freeman and Company, New York, 1990

[7] Lang. Linear Algebra. Columbia University, New York, 1980

[8] Olsder G.J. Mathematical System Theory. Deltse Uitgevers, Maatschappij, Netherlands, 1994

[9] Wonham W.M. Linear Multivariable Control: A Geometric Approach, Springer-Verlag, New York, 1979 